\title{
Le dépistage de la phénylcétonurie en France
}

> La phénylcétonurie (PCU) est la plus fréquente des erreurs innées du métabolisme et entraîne un retard mental irréversible en l'absence de traitement. Son dépistage néonatal a été rendu possible grâce à la technique de recueil de sang sur papier buvard mise au point par Robert Guthrie. Le dépistage néonatal de la PCU a débuté en France au début des années 1970. II a été initialement réalisé par une technique bactériologique, puis fluorimétrique et, enfin, depuis 2020 par spectrométrie de masse en tandem. Plus de 35 millions de nouveau-nés ont été dépistés à ce jour, ce qui a permis de diagnostiquer plus de 3500 enfants porteurs de PCU ou hyperphénylalaninémie modérée. La prise en charge de ces enfants a évolué avec le temps, en particulier grâce aux techniques de biochimie et de génétique moléculaire qui permettent un diagnostic précis et grâce à l'arrivée d'un traitement médicamenteux par saproptérine. Grâce à ce dépistage, qui permet une prise en charge précoce, le pronostic de la PCU a été transformé et, même s'il peut survenir des problèmes neurologiques ou comportementaux, ces patients ont une vie normale aujourd'hui. <

\section{Historique du dépistage néonatal de la phénylcétonurie}

La phénylcétonurie (PCU), la plus fréquente des erreurs innées du métabolisme $(\varepsilon I M)^{1}[1](\rightarrow)$ a été découverte en 1934 par Absjørn Følling [2], qui a mis en évidence la présence $(\rightarrow)$ Voir la Synthèse de A. Wiedemann et al., $m / s n^{\circ} 8-9$, août-septembre 2020 , page 725 d'acide phénylpyruvique dans les urines de patients présentant un retard mental. Le nom de phénylcétonurie a été donné par Penrose [3] en raison de la présence de phénylcétones dans les urines. La PCU a été la première maladie

Vignette (๔ CNCDN)

1 Ou maladies héréditaires du métabolisme.
Arnaud Wiedemann ${ }^{1,2}$, Élise Jeannesson ${ }^{2,3}$, Abderrahim Oussalah ${ }^{2,3}$, Jean-Louis Guéant ${ }^{2,3}$, Rosa-Maria Guéant-Rodriguez ${ }^{2,3}$, François Feillet ${ }^{1,2}$

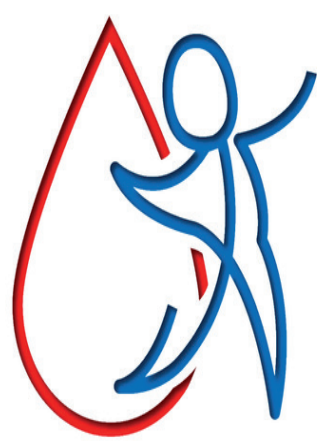

${ }^{1}$ Service de médecine infantile, Hôpital d'enfants, CHRU de Nancy, 54000 Nancy, France. ${ }^{2}$ Inserm UMR_S 1256 (NGERE, Nutrition génétique et exposition aux risques environnementaux), Faculté de médecine de Nancy, Université de Lorraine, 54000 Nancy, France.

${ }^{3}$ Département de médecine moléculaire, Laboratoire de biochimie et de biologie moléculaire nutrition, $\mathrm{CHRU}$ de Nancy, 54000 Nancy, France. f.feillet@chru-nancy.fr

métabolique traitable grâce à Horst Bickel [4], qui a mis en place, en 1953, un régime pauvre en phénylalanine, ce qui a permis d'améliorer considérablement le pronostic des patients. L'existence d'un traitement efficace a nourri la réflexion sur la nécessité d'un diagnostic précoce pour éviter la survenue du retard mental définitif lié à cette maladie. La première tentative de dépistage systématique de la PCU repose sur la proposition de Willard Centerwall d'ajouter du perchlorate de fer sur les couches des nouveau-nés contenant des urines fraîches, l'apparition d'une coloration verte foncée permettant en effet de faire le diagnostic [5]. Ce test n'était cependant pas fonctionnel au niveau d'une population entière, et c'est Robert Guthrie qui mit au point, en 1963, un test sur un échantillon de sang séché recueilli sur un carton buvard afin de permettre la réalisation généralisée de ce dépistage [6]. II est intéressant de noter que deux des personnes impliquées dans la découverte de la PCU et de son dépistage étaient touchées de près par cette maladie. Følling avait en effet deux enfants dans sa famille atteints de PCU et Guthrie était père d'un garçon handicapé et sa nièce était atteinte d'une PCU. La mise en place de ce dépistage a été la première étape de la grande histoire du dépistage néonatal qui aboutit aujourd'hui au dépistage de plus de 50 maladies différentes dans certains états des États-Unis.

\section{La phénylcétonurie}

La PCU est due à des mutations du gène PAH codant une enzyme hépatique, la phénylalanine hydroxylase (PAH) [7]. La PAH transforme la phénylalanine (Phé) en tyrosine en présence d'un cofacteur, la tétrahydrobioptérine (BH4) [8], et d'une molécule chaperonne DNAJC12 (Dnaj heat shock protein family [Hsp40] member C12) (Figure 1) [9]. La perte d'activité de la PAH entraîne un défaut du catabolisme de la phénylala- 


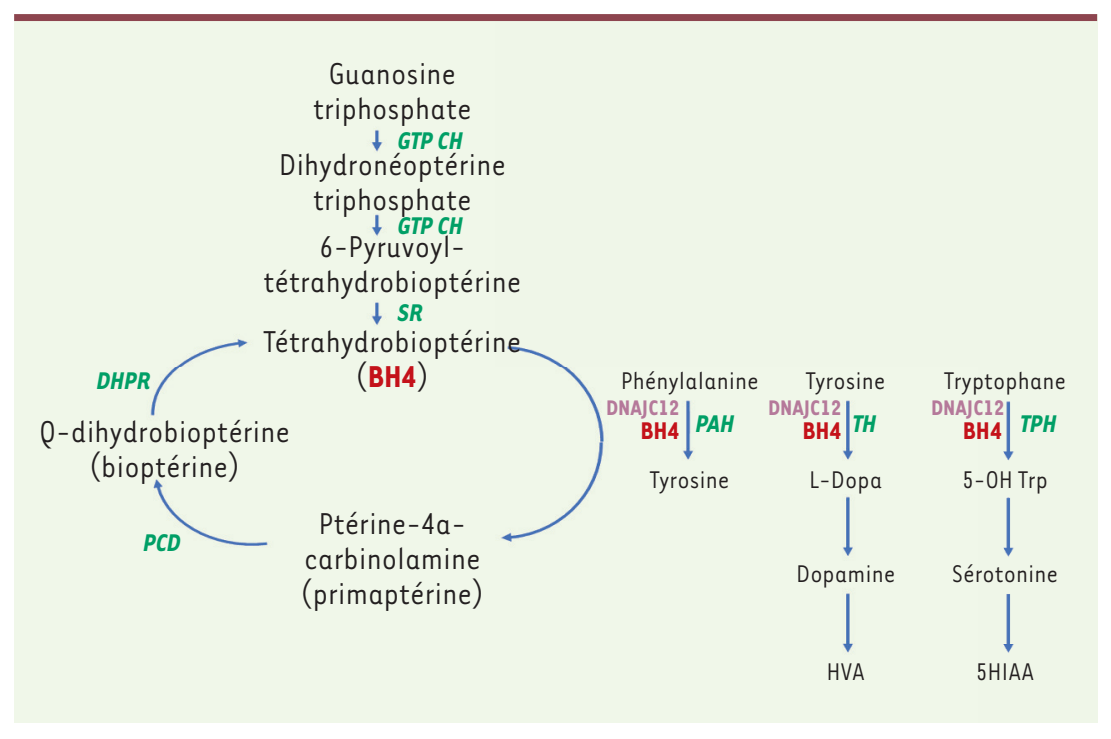

Figure 1. Voie métabolique de la phénylcétonurie. La phénylcétonurie est liée à un déficit de la phénylalanine hydroxylase (PAH) dont le fonctionnement nécessite la présence d'un cofacteur, la tétrahydrobioptérine (BH4). Ce cofacteur est également nécessaire au fonctionnement de la tyrosine hydroxylase (TH) et de la tryptophane hydroxylase (TPH). Un déficit en PAH entraîne une hyperphénylalaninémie isolée. Un déficit de synthèse (lié aux déficits en guanosine triphosphate cyclohydrolase [GTPCH], pyruvoyl-tétrahydrobioptérine synthase [PTPS] et sépiaptérine réductase $[S R]$ ) ou de recyclage (lié au déficit en ptérine-4a-carbinolamine déhydratase $[P C D]$ et dihydroptéridine réductase $[D H P R])$ de la BH4 (tétrahydrobioptérine) entraîne une hyperphénylalaninémie, mais également un déficit en certains neurotransmetteurs. Une molécule chaperonne (DNAJC12) est également nécessaire au bon fonctionnement de ces trois hydroxylases.

nine et donc une augmentation de la concentration plasmatique de cet acide aminé. La phénylalanine passe alors dans le cerveau au prorata de sa concentration plasmatique. Au-delà d'un certain seuil, elle provoque une atteinte neurologique sévère (retard mental irréversible, épilepsie, microcéphalie, troubles du spectre autistique, syndromes psychiatriques), associée à une dépigmentation cutanéo-phanérienne et à un eczéma [10]. La prise en charge de la maladie dès la période néonatale, que ce soit par un régime pauvre en phénylalanine ou par un traitement médicamenteux par saproptérine (version synthétique de la BH4), a permis aux enfants atteints d'avoir un devenir normal [11].

\section{Les techniques de dépistage de la phénylcétonurie}

La PCU est diagnostiquée en France par le dépistage néonatal depuis le début des années 1970 [12]. Le dépistage de la PCU est fondé sur le dosage de la phénylalanine sanguine entre 48 et 72 heures après la naissance [11]. Ce délai est indispensable car avant la naissance, la phénylalanine insuffisamment métabolisée par le fœtus atteint est en effet épurée par le foie de sa mère ; quelques jours sont alors nécessaires en post-natal avant de pouvoir détecter son accumulation chez le nouveau-né. Initialement, le dosage de la phénylalanine était réalisé par une technique bactériologique fondée sur la restauration de la croissance, en présence d'une tache de sang séché sur buvard, des bactéries d'une souche de Bacillus subtilis cultivées sur un milieu pauvre en phénylalanine; les diamètres des colonies bactériennes ayant crû sont alors proportionnels aux concentrations de phénylalanine contenues dans la tache de sang [6]. Cette technique a rapidement été remplacée par des techniques fluorimétriques [13] qui seront utilisées pendant une trentaine d'années jusqu'à ce que le dépistage évolue (enfin !) en France, le $1^{\text {er }}$ décembre 2020, avec l'extension du dépistage néonatal à d'autres maladies métaboliques, au premier rang desquelles le déficit en MCAD (acyl-coenzyme $A$ déshydrogénase des acides gras à chaîne moyenne) [14]. Le métabolite marqueur de ce dernier déficit, l'octanoylcarnitine, est désormais dosé conjointement à la phénylalanine par une technique de spectrométrie de masse en tandem qui permet la quantification simultanée de nombreux métabolites (acylcarnitines et acides aminés). Cette technique produit des taux de phénylalanine inférieurs de $30 \%$ à la technique fluorimétrique pour un même échantillon, ce qui a entraîné une diminution du seuil de dépistage positif, qui est passé de 180 à $120 \mu \mathrm{mol} / \mathrm{L}$.

\section{L’organisation du dépistage de la phénylcétonurie en France}

Le dépistage de la phénylcétonurie a été instauré en France à partir de 1966 grâce à des initiatives privées (à Lille, Paris et Lyon), puis en bénéficiant d'un soutien de la société Évian qui l'a promu. Ce dépistage a été généralisé sur l'ensemble du territoire français à partir de 1972. Le dépistage a ensuite été géré par l'Association française pour le dépistage des handicaps de l'enfant (AFDPHE) à partir de 1978. Le financement du dépistage a été assuré par la CNAMTS (Caisse nationale d'assurance maladie des travailleurs salariés). En 2018, l'organisation a été remaniée et dépend maintenant de la Direction générale de la santé (DGS) à travers les Agences régionales de santé. Depuis 1970, plus de 35 millions d'enfants ont bénéficié de ce dépistage. II a permis de dépister 1895 enfants porteurs de PCU et 2037 porteurs d'hyperphénylalaninémie modérée permanente (HPM). 


\section{Aspects pratiques du dépistage de la PCU}

Le dépistage de la PCU est fondé sur le dosage par LC/MSMS (liquid chromatography coupled to tandem mass spectrometry) de la phénylalanine sur un carton buvard. Un dépistage est dit positif si la concentration de phénylalanine est supérieure à $2 \mathrm{mg} / \mathrm{dL}$ ou $120 \mu \mathrm{mol} / \mathrm{L}$. Ce seuil a été actualisé récemment en raison du changement de la méthode de dosage; il était de $3 \mathrm{mg} / \mathrm{dL}$ ou $180 \mu \mathrm{mol} / \mathrm{L}$ lorsque le dosage était réalisé par fluorimétrie. Le seuil de $120 \mu \mathrm{mol} / \mathrm{L}$ correspond aux recommandations actuelles, à la fois américaine et européenne $[15,16]$. Lorsque la concentration de phénylalanine est supérieure à $120 \mu \mathrm{mol} / \mathrm{L}(2 \mathrm{mg} / \mathrm{dL})$, un prélèvement de contrôle est réalisé. Si la concentration de contrôle est inférieure à $120 \mu \mathrm{mol} / \mathrm{L}$, le dépistage est classé comme faux positif. En revanche, si la concentration de phénylalanine reste supérieure à $120 \mu \mathrm{mol} / \mathrm{L}$, le dépistage est considéré comme positif et le nouveau-né rejoint le groupe des enfants pris en charge médicalement [11]. Cette prise en charge comportera l'élimination des diagnostics différentiels et la confirmation du diagnostic de PCU (aminogramme plasmatique, génotypage et étude de la sensibilité à la BH4).

\section{Prise en charge initiale du nouveau-né dépisté}

$\varepsilon$ n cas de dépistage positif, le centre de dépistage néonatal prévient le médecin référent de la zone géographique concernée pour une prise en charge immédiate de l'enfant qui bénéficiera alors des examens suivants : - Contrôle de la concentration de phénylalanine sur un nouveau buvard de sang séché,

- Bilan hépatique et chromatographie des acides aminés plasmatiques pour éliminer les autres causes d'hyperphénylalaninémie,

- Profil des ptérines urinaires et mesure de l'activité dihydroptéridine réductase (DHPR) sanguine pour dépister une anomalie du métabolisme de BH4. Ces prélèvements doivent être réalisés avant la prise de BH4 pour le test néonatal,

- Génotypage,

- Test de sensibilité à la BH4 si le taux de contrôlé est supérieur à $360 \mu \mathrm{mol} / \mathrm{L}(6 \mathrm{mg} / \mathrm{dL})$ [17] qui est le seuil de traitement du patient.

Ces examens sont le plus souvent réalisés au cours d'une hospitalisation ou, en fonction de la gravité de l'augmentation de la concentration de phénylalanine et selon l'organisation locale, en hôpital de jour ou en consultation externe. Ils doivent être réalisés en urgence (idéalement, les nouveau-nés devraient être vus le jour même du dépistage positif et de l'appel téléphonique) si le taux est supérieur à $360 \mu \mathrm{mol} / \mathrm{L}$ $(6 \mathrm{mg} / \mathrm{dL})$ et sans urgence dans le cas contraire. L'annonce du diagnostic comprendra une information sur la maladie, sa prise en charge et les modalités de l'éducation thérapeutique qui sera mise en place.

\section{Diagnostics différentiels des hyperphénylalaninémies néonatales}

\section{Les maladies héréditaires du métabolisme}

Les déficits du métabolisme de la BH4. Les déficits de synthèse ou de recyclage de la BH4 doivent être systématiquement recherchés devant toute hyperphénylalaninémie (néonatale ou non) par l'étude des ptérines urinaires et de l'activité sanguine des DHPR (récepteurs des dihydropyridines). Ces déficits entraînent une augmentation de la phénylalanine, mais également des déficits en neurotransmetteurs cérébraux (déficits de synthèse de la $\mathrm{BH} 4$ ) et une déplétion secondaire en folates cérébraux (déficit en DHPR) [18]. Ces déficits nécessitent un diagnostic et un traitement urgents.

Le déficit en DNAJC12. Un nouveau déficit pouvant entraîner une hyperphénylalaninémie a été décrit en 2017. DNAJC12 (DnaJ heat shock protein family (Hsp40) member $(12)$ est une molécule chaperonne qui stabilise Ia PAH ainsi que les autres hydroxylases responsables de la synthèse de certains neurotransmetteurs [9]. Les patients déficitaires nécessitent un traitement par la $\mathrm{BH} 4$ et par neurotransmetteurs. Le diagnostic se fait par analyse moléculaire du gène DNAJC12 après avoir éliminé les autres causes d'hyperphénylalaninémie.

Les autres maladies héréditaires du métabolisme. II s'agit essentiellement des maladies entraînant une insuffisance hépatique: galactosémie, fructosémie, tyrosinémie et déficits de la chaîne respiratoire [11].

\section{Les causes non métaboliques}

Le diagnostic différentiel est fondé sur le bilan hépatique et sur l'aminogramme plasmatique :

- Hyperphénylalaninémie transitoire (prématuré +++),

- Perfusion d'acides aminés,

- Insuffisance hépatocellulaire néonatale, quelle qu'en soit la cause (sepsis, hémochromatose néonatale ou autre),

- Hyperphénylalaninémie secondaire à un médicament (triméthoprime, méthotrexate, antifoliques).

\section{Efficacité du dépistage néonatal de la PCU et fréquence des patients PCU en France au cours du temps}

\section{Données globales}

Depuis la mise en place du dépistage néonatal de la phénylcétonurie en 1972, plus de 35 millions de nouveau-nés ont été dépistés [19]. D'après les chiffres du rapport d'activité du Centre national de dépistage néonatal de 2018, ce dépistage a permis d'identifier 2125 patients PCU et 1448 patients HPM [19]. La grande majorité des patients sont issus de la Métropole, où la fréquence de la PCU est de 1/15908 alors qu'elle n'est que de 1/77034 dans les départements et territoires d'outre-mer. La fréquence des HPM est de 1/23159 en Métropole; elle n'est que de $1 / 47860$ outre-mer avec une disparité ethnique importante, car aucun cas n'a été dépisté à Mayotte $(0 / 154780)$ et un seul cas en 


\begin{tabular}{|c|c|c|c|}
\hline Région & Fréquence PCU & Fréquence HPM & $\begin{array}{l}\text { Fréquence } \\
\text { PCU + HPM }\end{array}$ \\
\hline Auvergne-Rhône-Alpes & $1 / 17867$ & $1 / 18314$ & $1 / 9044$ \\
\hline Bourgogne-Franche-Comté & $1 / 12106$ & $1 / 22835$ & $1 / 7875$ \\
\hline Bretagne & $1 / 21918$ & $1 / 65754$ & $1 / 16439$ \\
\hline Centre-Val-de-Loire & $1 / 20210$ & $1 / 61820$ & $1 / 15231$ \\
\hline Grand Est & $1 / 11618$ & $1 / 20790$ & $1 / 7453$ \\
\hline Hauts-de-France & $1 / 12711$ & $1 / 25422$ & $1 / 8477$ \\
\hline Île-de-France & $1 / 18064$ & $1 / 17631$ & $1 / 8923$ \\
\hline Normandie & $1 / 12707$ & $1 / 43470$ & $1 / 9832$ \\
\hline Nouvelle Aquitaine & $1 / 17913$ & $1 / 29286$ & $1 / 11115$ \\
\hline Occitanie & $1 / 17792$ & $1 / 21865$ & $1 / 9810$ \\
\hline PACA-Corse & $1 / 13868$ & $1 / 22671$ & $1 / 8605$ \\
\hline Pays de Loire & $1 / 24685$ & $1 / 33218$ & $1 / 14161$ \\
\hline DOM-TOM & $1 / 77034$ & $1 / 119052$ & $1 / 46771$ \\
\hline Total & $1 / 16397$ & $1 / 23881$ & $1 / 9722$ \\
\hline
\end{tabular}

Tableau I. Fréquence de la PCU et des hyperphénylalaninémies modérées dans les différentes régions françaises de 1978 à 2018 . La fréquence de la PCU varie du simple au double en fonction des régions et est beaucoup plus rare dans les départements et territoires d'outre-mer [19]. PCU : phénylcétonurie ; HPM : hyperphénylalaninémie modérée.

Guyane (1/179577), ce qui conduit sur ces deux territoires à une fréquence PCU + HPM de 1/330 357 nouveau-nés dépistés (Tableau I). En Métropole, une disparité de fréquence de la PCU est également observée, celle-ci pouvant varier du simple au double (Tableau I).

Il faut noter également que ce dépistage a permis d'identifier des déficits touchant la synthèse ou le recyclage du cofacteur de la PAH, la tétrahydrobioptérine (ou BH4), et que l'on a dénommés formes malignes de $P C U$, ceux-ci ne répondant pas au traitement diététique classique $[20,21]$. Cinquante et un patients porteurs de déficit du métabolisme de la BH4 ont pu être diagnostiqués de 1972 à 2018, ce qui correspond à une fréquence très faible ( $1,4 \%$ des patients dépistés pour hyperphénylalaninémie), conforme à ce qui est décrit dans la littérature [22].

\section{Évolution au cours du temps}

Le dépistage de la PCU a demandé une dizaine d'années pour atteindre son rythme de croisière (Figure 2), avec une stabilisation du nombre de patients PCU dépistés après 10 ans d'activité. Par contre, une augmentation du nombre de patients HPM dépistés a été observée à partir des années 1980. Cette augmentation est liée au passage de la méthode bactériologique à la méthode fluorimétrique pour ce dépistage. Cette dernière méthode étant plus sensible, il est normal que le nombre de patients HPM dépistés ait augmenté. Le seuil du dépistage a également été abaissé (de 180 à $120 \mu \mathrm{mol} / \mathrm{L}$ ) à l'occasion du passage, en 2020, à la méthode fondée sur la spectrométrie de masse en tandem. Il est néanmoins encore trop tôt pour déterminer si cette modification de dosage, et donc des seuils, aura pour effet une augmentation (ou non) du nombre de nouveau-nés dépistés.

\section{Efficacité globale du dépistage de la PCU}

Selon les données de l'AFDPHE (rapport d'activité 2017), la valeur prédictive positive (VPP : nombre de vrais positifs sur le nombre de tests positifs) est élevée. Elle est de 19,4\% pour l'ensemble des hyperphénylalaninémies et de $8,6 \%$ pour les PCU. Les faux positifs sont essentiellement constitués de nouveau-nés hospitalisés pour prématurité avec plus ou moins de maladies associées (sepsis, insuffisance hépatique, nutrition parentérale, etc.) ou porteurs d'autres maladies métaboliques, principalement la galactosémie [23] ou la tyrosinémie de type I. Le dépistage de la PCU ne comporte que très peu de faux négatifs. Les diagnostics tardifs de PCU sont essentiellement liés aux patients issus de pays où le dépistage néonatal de la PCU n'est pas réalisé de façon systématique (enfants issus de l'immigration essentiellement) [10]. 

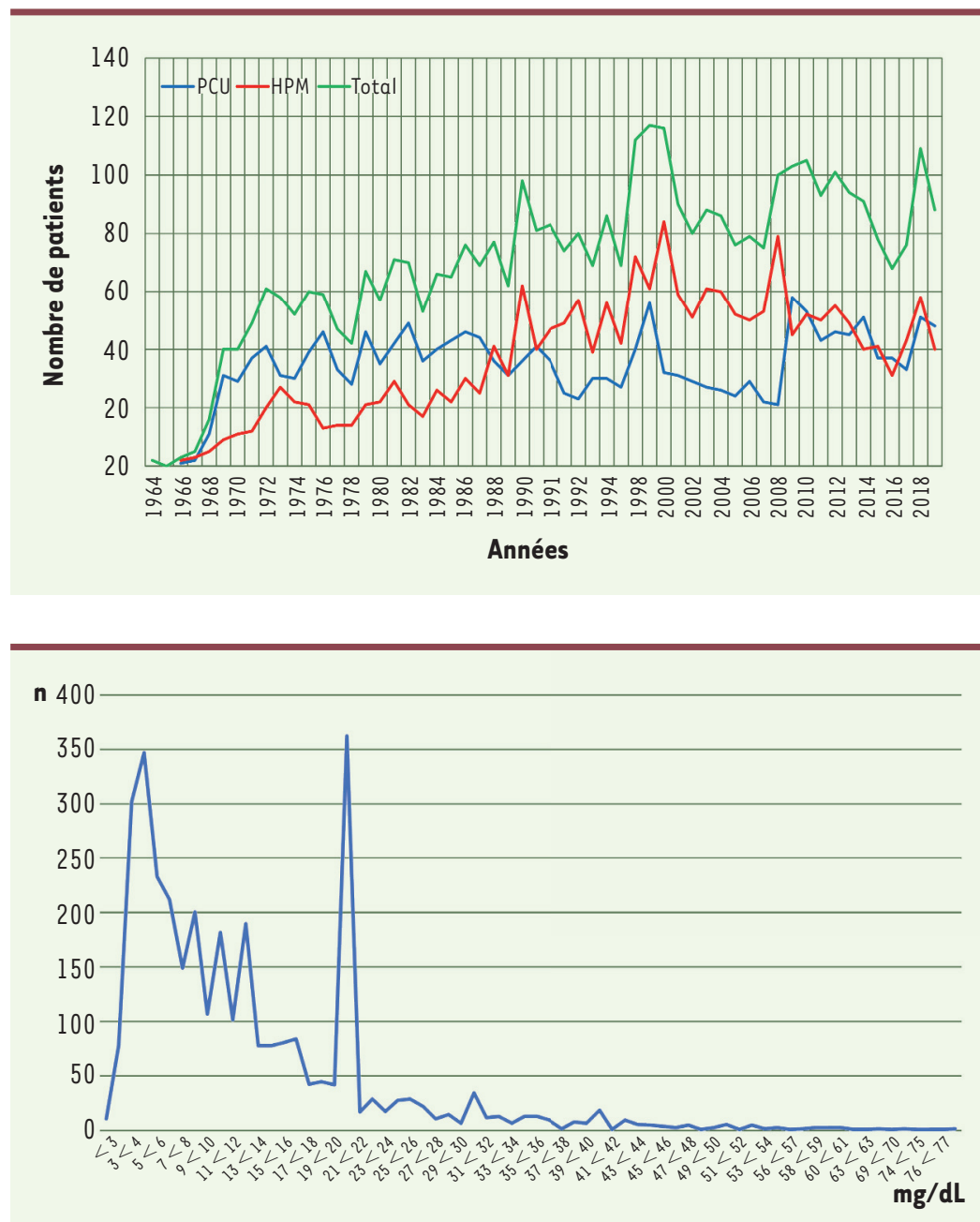

Figure 2. Nombre de patients porteurs d'hyperphénylalaninémie, dépistés par an depuis le début du dépistage néonatal en France. PCU : phénylcétonurie; HPM : hyperphénylalaninémie modérée; Total: PCU + HPM. Ces données sont issues de celles de l'AFDPHE et, pour les données depuis 2018, du Centre national de coordination du dépistage néonatal (CNCDN).

Figure 3. Concentrations de la phénylalanine sanguine à $J 3$ (en mg/dL) chez les 3321 nouveau-nés dépistés positifs depuis le début du dépistage en France. $\mathrm{n}$ : nombre de patients; $\mathrm{mg} / \mathrm{dL}$ : unité de dosage de la phénylalanine.
À noter que le prix d'une analyse par fluorimétrie était de $1,98 €$; le coût global était donc d'environ 1,54 million d'euros par an, soit un coût par malade repéré de $45188 €$ (pour les 34 PCU) ou de $19953 €$ (en ajoutant les 43 HPM).

\section{Résultats du dépistage néonatal, génétique, et réponse à la BH4}

Grâce aux données collectées par l'AFDPHE, il a été possible d'établir la répartition des concentrations de phénylalanine au dépistage (dosée par méthode fluorimétrique) sur plus de 3000 nouveau-nés dépistés pour hyperphénylalaninémie (Figure 3 ). En fonction de ces concentrations de phénylalanine, on peut considérer 3 groupes d'enfants. Dans le premier groupe, le plus grand, les nouveau-nés avaient un taux de phénylalanine aux alentours du seuil de dépistage (soit $3 \mathrm{mg} / \mathrm{dL}$ ou $180 \mu \mathrm{mol} / \mathrm{L}$ ). Ces enfants n'ont pas été traités pendant des décennies; les recommandations françaises de traiter au-dessus de $6 \mathrm{mg} / \mathrm{dL}$ (ou $360 \mu \mathrm{mol} / \mathrm{L}$ ) ne datent en effet que de 2018 [11]. Un certain nombre de ces enfants sont hétérozygotes pour des variants pathogènes du gène $P A H$, comme cela a été montré dans une étude française réalisée sur plus de 700 génotypes de patients PCU [24]. Le deuxième groupe comprend les patients dont les taux sont entre 6 et $15 \mathrm{mg} / \mathrm{dL}$ (360 et $900 \mu \mathrm{mol} / \mathrm{L}$ ). Ces enfants, qui ont des formes partielles de PCU, seront les plus susceptibles d'être traités par la $\mathrm{BH} 4$ [17]. Le troisième groupe est constitué des enfants ayant des concentrations de phénylalanine aux alentours de $20 \mathrm{mg} / \mathrm{dL}$ (ou $1200 \mu \mathrm{mol} / \mathrm{L}$ ). Ces enfants sont majoritairement ceux qui portent deux mutations sévères du gène entraînant une activité $\mathrm{PAH}$ nulle. Les quelques enfants qui ont des taux supérieurs à $20 \mathrm{mg} / \mathrm{dL}$ correspondent à ceux qui ont deux mutations sévères et qui avaient probablement un état de catabolisme important qui a majoré le taux de phénylalanine à J3.

\section{Devenir des patients phénylcétonuriques}

Le devenir des patients PCU a été radicalement modifié grâce au dépistage néonatal. Avant sa mise en place, les patients présentaient un retard mental et un tableau neurologique sévère dont la gravité était proportionnelle au retard du diagnostic [15].

Initialement, les traitements étaient arrêtés pendant l'enfance ou au début de l'adolescence. L'apparition de complications neurologiques $[25,26]$ et/ou psychia- 
triques [27] chez ces patients ayant arrêté leur régime, a conduit à une évolution de leur prise en charge. Ces atteintes sont réversibles après un retour à un contrôle métabolique strict. II est maintenant recommandé de poursuivre le régime toute la vie durant afin d'éviter les complications à long terme chez ces patients [15]. Grâce à ces recommandations, le devenir des patients est très bon : ils accomplissent leurs études et s'insèrent dans une vie professionnelle et familiale proche de la normale. Une étude est actuellement en cours pour évaluer les comorbidités qui surviennent chez les patients PCU en France, comme cela a été réalisé aux États-Unis [28] et en Allemagne [29]. Cette étude permettra de déterminer l'efficacité de la prise en charge dans notre pays, celle-ci étant liée au dépistage, mais également à la qualité des centres spécialisés et du système national de soins.

\section{Conclusion}

La phénylcétonurie est à l'origine de la mise en place du dépistage néonatal, grâce à plusieurs médecins et biologistes qui ont découvert la maladie, puis les moyens de la traiter et de la dépister à l'échelle d'une population. Cela a été le début de la merveilleuse histoire du dépistage néonatal, qui permet désormais, chaque année et dans le monde entier, la prise en charge en pré-symptomatique de milliers d'enfants, évitant ainsi les conséquences délétères des maladies dépistées. $\diamond$

\section{SUMMARY}

\section{Newborn screening of phenylketonuria in France}

Phenylketonuria is the most common inborn error of metabolism and causes irreversible mental retardation if left untreated. Its newborn screening was made possible by the technique of blood collection on filter paper developed by Robert Guthrie. Neonatal PKU screening began in France in the early 1970s. It was initially carried out by a bacteriological method, then by fluorometry, and finally, since 2020 , by tandem mass spectrometry. More than 35 million newborns have been screened to date. This resulted in the diagnosis of more than 3,500 children with PKU or mild hyperphenylalaninemia. The management of these children has improved over time, in particular thanks to the techniques of biochemistry and molecular genetics which lead to an accurate diagnosis and the arrival of drug treatment by sapropterin. Thanks to this screening, which allows for early management, the prognosis of PKU has been transformed and, although neurological or behavioral problems may arise, these patients are living normally today. $\diamond$

\section{LIENS D'INTÉRÊT}

Les auteurs déclarent n'avoir aucun lien d'intérêt concernant les données publiées dans cet article.

\section{RéFÉRENCES}

1. Wiedemann $A$, Oussalah $A$, Jeannesson $\varepsilon$, et al. La phénylcétonurie : de la diététique à la thérapie génique. Med Sci (Paris) $2020 ; 36: 725-34$.

2. Folling I. The discovery of phenylketonuria. Acta Paediatr $1994 ; 407$ (suppl) : 4-10.

3. Penrose L, Quastel JH. Metabolic studies in phenylketonuria. Biochem J $1937 ; 31: 266-74$.

4. Bickel H. Diagnosis and therapy of galactosemia and phenylketonuria. Monatsschrift Kinderheilkunde $1955 ; 103: 81-4$.
5. Centerwall WR. Phenylketonuria. J Am Med Assoc 1957 ; 165 : 392.

6. Guthrie R, Susi A. A simple phenylalanine method for detecting phenylketonuria in large populations of newborn infants. Pediatrics 1963 ; $32: 338-43$.

7. Woo SL, Lidsky AS, Guttler F, et al. Cloned human phenylalanine hydroxylase gene allows prenatal diagnosis and carrier detection of classical phenylketonuria. Nature $1983 ; 306: 151-5$.

8. Danks DM, Cotton RG, Schlesinger P. Letter: Tetrahydrobiopterin treatment of variant form of phenylketonuria. Lancet $1975 ; 2$ : 1043.

9. Anikster Y, Haack TB, Vilboux T, et al. Biallelic mutations in DNAJC12 cause hyperphenylalaninemia, dystonia, and intellectual disability. Am J Hum Genet 2017; $100: 257-66$.

10. Van Wegberg AMJ, MacDonald A, Ahring K, et al. The complete European guidelines on phenylketonuria: diagnosis and treatment. Orphanet J Rare Dis 2017; $12: 162$.

11. HAS. Plan National de Diagnostic et de Soins. Phénylcétonurie. https://www. has-sante.fr/upload/docs/application/pdf/2018-06/phenylcetonurie_-_ pnds.pdf. 2018.

12. Abadie V, Berthelot J, Feillet F, et al. Neonatal screening and long-term follow-up of phenylketonuria: the French database. Early Hum Dev 2001; $65: 149-58$.

13. Searle B, Mijuskovic MB, Widelock D, Davidow B. A manual fluorometric paper disc method for detecting phenylketonuria. Clin Chem 1967; 13:6215.

14. HAS. https://www.has-sante.fr/upload/docs/application/pdf/2011-07/ argu_depistage_neonatal_vf.pdf. 2011.

15. van Spronsen FJ, van Wegberg AM, Ahring K, et al. Key European guidelines for the diagnosis and management of patients with phenylketonuria. Lancet Diabetes Endocrinol 2017; 5 : 743-56.

16. Camp KM, Parisi MA, Acosta PB, et al. Phenylketonuria scientific review conference: state of the science and future research needs. Mol Genet Metab 2014; $112: 87-122$.

17. Muntau AC, du Moulin M, Feillet F. Diagnostic and therapeutic recommendations for the treatment of hyperphenylalaninemia in patients 0-4 years of age. Orphanet J Rare Dis 2018; $13: 173$.

18. Blau N, Dhondt JL. Tetrahydrobiopterin deficiency and an international database of patients. Adv Exp Med Biol 1993; 338 : 255-61.

19. CNCDN. http://depistage-neonatal.org/wp-content/uploads/2019/12/ Rapport-Activite-2018.pdf. 2020.

20. Kaufman S, Berlow S, Summer GK, et al. Hyperphenylalaninemia due to a deficiency of biopterin. A variant form of phenylketonuria. N Engl J Med 1978; $299: 673-9$.

21. Blau N, Thony B, Spada M, Ponzone A. Tetrahydrobiopterin and inherited hyperphenylalaninemias. Turk J Pediatr 1996 ; 38 : 19-35.

22. Blau N, Barnes I, Dhondt JL. International database of tetrahydrobiopterin deficiencies. J Inherit Metab Dis 1996; $19:$ 8-14.

23. Shakespeare L, Downing $M$, Allen J, et al. Elevated phenylalanine on newborn screening: follow-up testing may reveal undiagnosed galactosaemia. Ann Clin Biochem 2010; $47: 567-9$.

24. Jeannesson-Thivisol $\varepsilon$, Feillet F, Chery C, et al. Genotype-phenotype associations in French patients with phenylketonuria and importance of genotype for full assessment of tetrahydrobiopterin responsiveness. Orphanet J Rare Dis 2015; $10: 158$.

25. Seki M, Takizawa T, Suzuki S, et al. Adult phenylketonuria presenting with subacute severe neurologic symptoms. J Clin Neurosci 2015; 22 : 1361-3.

26. Rubin S, Piffer AL, Rougier MB, et al. Sight-threatening phenylketonuric encephalopathy in a young adult, reversed by diet. JIMD Reports 2013; 10 : 83-5.

27. Ashe K, Kelso W, Farrand S, et al. Psychiatric and cognitive aspects of phenylketonuria: the limitations of diet and promise of new treatments. Front Psychiatry 2019; $10: 561$.

28. Burton BK, Jones KB, Cederbaum S, et al. Prevalence of comorbid conditions among adult patients diagnosed with phenylketonuria. Mol Genet Metab 2018; 125 : 228-34.

29. Trefz KF, Muntau AC, Kohlscheen KM, et al. Clinical burden of illness in patients with phenylketonuria (PKU) and associated comorbidities: a retrospective study of German health insurance claims data. Orphanet J Rare Dis 2019; 14 : 181.
TIRÉS À PART

F. Feillet 